\title{
Regioselective (thio)carbamoylation of 2,7-di-tert-butylpyrene at the 1-position with iso(thio)cyanates
}

\author{
Anna Wrona-Piotrowicz ${ }^{* 1}$, Marzena Witalewska ${ }^{1}$, Janusz Zakrzewski ${ }^{* 1}$ and Anna Makal ${ }^{2}$
}

\section{Full Research Paper}

\section{Address:}

${ }^{1}$ Department of Organic Chemistry, Faculty of Chemistry, University of Łódź, Tamka 12, 91-403 Łódź, Poland and 2University of Warsaw, Biological and Chemical Research Center, Żwirki i Wigury 101, 02-089 Warszawa, Poland

Email:

Anna Wrona-Piotrowicz - awronapiotrowicz@gmail.com;

Janusz Zakrzewski* - janzak@uni.lodz.pl

${ }^{*}$ Corresponding author

Keywords:

amide; Friedel-Crafts; isocyanate; isothiocyanate; pyrene; thioamide
Beilstein J. Org. Chem. 2017, 13, 1032-1038.

doi:10.3762/bjoc. 13.102

Received: 07 March 2017

Accepted: 05 May 2017

Published: 29 May 2017

Associate Editor: B. Stoltz

๑) 2017 Wrona-Piotrowicz et al.; licensee Beilstein-Institut. License and terms: see end of document.

\begin{abstract}
It has been found that 2,7-di-tert-butylpyrene reacts with aliphatic iso(thio)cyanates in the presence of trifluoromethanesulfonic acid to exclusively afford the corresponding 1-substituted (thio)amides in high yields. For aromatic iso(thio)cyanates the reaction is less regioselective, although substitution at the 1-position prevails. For ethoxycarbonyl isothiocyanate, apart from the 1-substituted thioamide, 1,8-disubstituted thioamide and 2,7-di-tert-butylpyrene-1-carbonitrile are formed (especially at longer reaction times).
\end{abstract}

\section{Introduction}

Direct functionalization of pyrene $\mathbf{1}$ has attracted a great deal of attention in recent years because it is the most straightforward route to novel organic molecular materials for optoelectronic devices (OLEDs, field-effect transistors, fluorescent sensors, dye lasers, etc.) [1-7]. Since 1 is an electron-rich arene, aromatic electrophilic substitution seems to be the simplest method for this purpose, and a plethora of substituted pyrenes have been synthesized in this way [1]. As is shown in Figure 1, the most reactive in such reactions are positions 1, 3, 6 and 8 of 1 . However, Friedel-Crafts alkylation of $\mathbf{1}$ with an excess of sterically hindered tert-butyl chloride leads to 2,7-di-tert-butylpyrene (2) [8]. This compound, owing to the presence of two bulky and electron-donating tert-butyl groups, displays different reactivity towards electrophiles. It has been reported that nitration and bromination of 2 take place at the 1-position (however, the bromine atom in 1-bromopyrene can migrate into the 4-position in the presence of $\mathrm{AlCl}_{3}$ ) [8,9], whereas Friedel-Crafts acylation and Vilsmeier formylation take place at the 4-position [10].

We recently reported an efficient method for the synthesis of pyrene-1-carbothioamides via reaction of pyrene with isothiocyanates in the presence of trifluoromethanesulfonic (triflic) acid (TfOH) $[11,12]$. Since 2 was used as a starting material in 


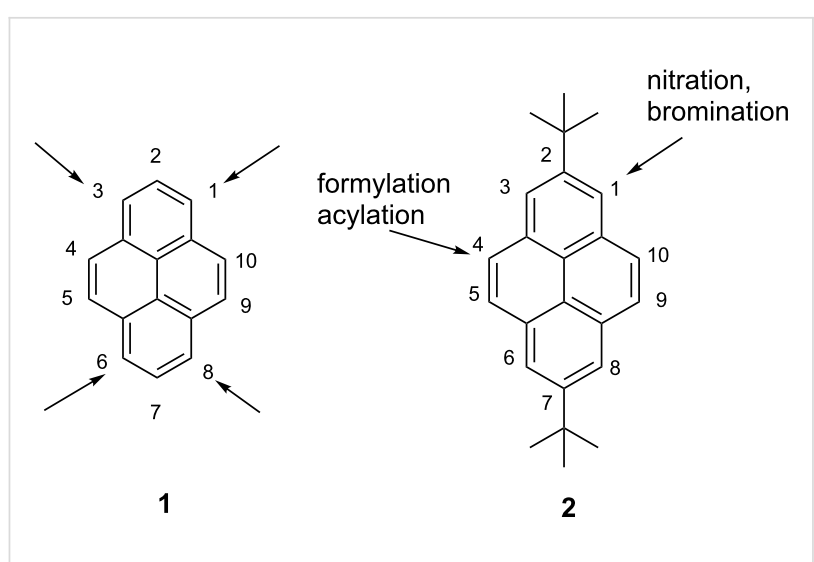

Figure 1: Sites of electrophilic attack in $\mathbf{1}$ and 2.

the syntheses of various pyrenyl fluorophores exhibiting unique photophysical properties [4,10,13-20], we thought it would be of interest to study its reactivity in the above reaction (and to extend its scope for isocyanates). An additional reason for such a study was the fact that thioamides (and amides) are versatile starting materials in the syntheses of various products (especially heterocycles) [21,22]. Furthermore, aromatic amides undergo a variety of directed $\mathrm{C}-\mathrm{H}$ bond functionalizations [23] Herein we report that the reaction of $\mathbf{2}$ with aliphatic isocyanates and isothiocyanates proceeds regioselectively at the 1-position (Scheme 1).

\section{Results and Discussion}

The reaction was carried out at room temperature in dichloromethane using 1.7 equiv of isocyanate or isothiocyanate and 3.3 equiv of TfOH. For the isocyanates, the reaction was completed in less than $5 \mathrm{~min}$, whereas the reaction with isothiocyanates required $\approx 30 \mathrm{~min}$ for completion. For the aliphatic isocyanates or isothiocyanates it afforded exclusively C1-substituted (thio)amides (as deduced from the splitting pattern of the pyrenyl protons in their ${ }^{1} \mathrm{H}$ NMR spectra, containing one pair of 1 -proton doublets displaying a meta-coupling constant $(\approx 2 \mathrm{~Hz}$, $\mathrm{H} 6$ and $\mathrm{H} 8$ ), two pairs of 1-proton doublets displaying orthocoupling constants $(\approx 7 \mathrm{~Hz}, \mathrm{H} 4, \mathrm{H} 5$ and $\mathrm{H} 9, \mathrm{H} 10)$ and one 1-proton singlet (H3).

Unexpectedly, the reaction of $\mathbf{2}$ with aromatic iso(thio)cyanates was less selective and led to inseparable mixtures of 1-subtituted (main components) and 4-substituted products $\mathbf{3 e , m , n}$ However, for the reaction with $p$-methoxyphenyl isothiocyanate we were able to obtain, by crystallization, the major product, $\mathrm{C}(1)$-substituted thioamide $\mathbf{3 n}$, contaminated with only $\approx 5 \%$ of its $\mathrm{C} 4$-substituted counterpart. We tentatively explain this poorer regioselectivity by possible $\pi-\pi$ interaction of pyrene with the arene ring of the protonated aromatic iso(thio)cyanate during formation of the Wheland complex, which may direct the electrophilic attack partly to the 4-position of the pyrene moiety.

To the best of our knowledge, the reaction reported here is the first example of a $\mathrm{C} 1$-selective substitution of 2 leading to the formation of a $\mathrm{C}-\mathrm{C}$ bond.

The presence of two tert-butyl groups significantly increases the reactivity of $\mathbf{2}$ in comparison with $\mathbf{1}$. A control experiment in which an equimolar mixture of $\mathbf{1}$ and $\mathbf{2}$ was treated in dichloromethane at $\mathrm{rt}$ with one equiv of tert-butyl isothiocyanate and 2 equiv of TfOH for $30 \mathrm{~min}$ resulted in practically exclusive formation of thioamide 3d (the amount of the product of thiocarbamoylation of 1 was estimated as $<2 \%$ ).

The reaction of $\mathbf{2}$ with ethoxycarbonyl isothiocyanate ( 2 equiv) in the presence of TfOH ran in a more complex way (Scheme 2, Table 1).

After $10 \mathrm{~min}, 1,8$-bis-carbothioamide 4 , containing $\approx 20 \%$ of other regioisomers $(9 \%)$ and nitrile 5 (7\%) were isolated by column chromatography besides the expected 30 (78\%). The amounts of $\mathbf{4}$ and $\mathbf{5}$ significantly increased along with the reaction time (Table 1). The highest yield of 4 (33\%) was found after $6 \mathrm{~h}$, whereas a 7-day reaction afforded $\mathbf{5}$ in a moderate $(54 \%)$ isolated yield.

Attempts to separate the regioisomeric bis-thioamides failed, but repeated chromatography and recrystallization from dichloromethane/hexane allowed isolation of the practically pure main regioisomer, 1,8-dithioamide 4. Its structure was confirmed by single-crystal $\mathrm{X}$-ray diffraction, which revealed a cisoidal conformation of this molecule in the crystal (Figure 2, for details, see Supporting Information File 1 and Supporting Information File 2). However, the ${ }^{1} \mathrm{H}$ NMR spectrum of 4 at room temperature was more complex than expected and contained broadened signals, suggesting the presence of other rotamers in $\mathrm{CDCl}_{3}$ solution.

The mechanism of the formation of nitrile 5 in the reaction under study here is so far unclear. The control experiment showed that under reaction conditions the secondary thioamide 30 undergoes transformation into $\mathbf{5}$, but to our knowledge this reaction, which must involve cleavage of the $\mathrm{C}-\mathrm{N}$ bond, has no precedent in the literature (in contrast to the well-known formation of nitriles from primary thioamides, involving formal elimination of $\left.\mathrm{H}_{2} \mathrm{~S}[24]\right)$.

Encouraged by the above results, we decided to check whether TfOH-promoted Friedel-Crafts acylation of $\mathbf{2}$ will also occur at the 1-position. For this purpose, we examined the reaction of 2 


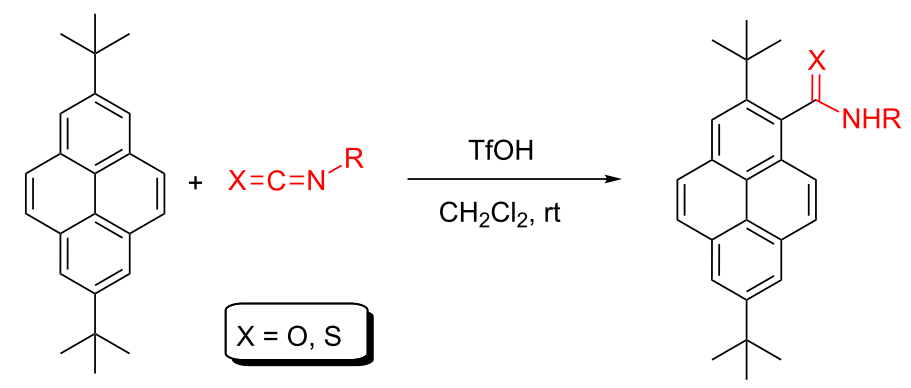

2

$3 a-n$<smiles>CCNC(=O)c1c(C(C)(C)C)cc2ccc3cc(C(C)(C)C)cc4ccc1c2c34</smiles>

$3 \mathbf{a}(76 \%)$

3b (64\%)<smiles>CC(C)(C)c1cc2ccc3cc(C(C)(C)C)c(C(=O)NCl)c4ccc(c1)c2c34</smiles>

$3 c(72 \%)$<smiles>CCCCNC(=O)c1c(C(C)(C)C)cc2ccc3cc(C(C)(C)C)cc4ccc1c2c34</smiles>

3d $(84 \%)$

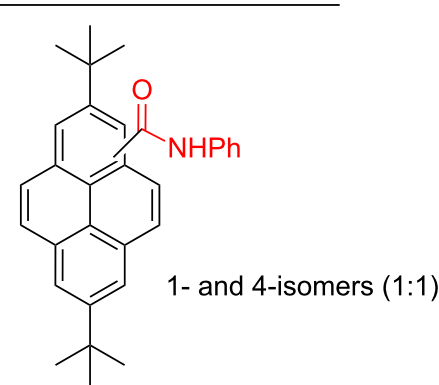

$3 e(63 \%)$<smiles>CCNC(=S)c1c(C(C)(C)C)cc2ccc3cc(C(C)(C)C)cc4ccc1c2c34</smiles>

$3 f(94 \%)$<smiles>CCNC(=O)c1c(C(C)(C)C)cc2ccc3cc(C(C)(C)C)cc4ccc1c2c34</smiles>

3g $(89 \%)$<smiles>CCCCNC(=O)c1c(C(C)(C)C)cc2ccc3cc(C(C)(C)C)cc4ccc1c2c34</smiles>

3h (91\%)<smiles>CCCCCNC(=S)c1c(C(C)(C)C)cc2ccc3cc(C(C)(C)C)cc4ccc1c2c34</smiles>

$3 \mathbf{i}(93 \%)$<smiles>CCCCCCCCNC(=S)c1c(C(C)(C)C)cc2ccc3cc(C(C)(C)C)cc4ccc1c2c34</smiles>

3j (87\%)<smiles>CC(C)(C)c1cc2ccc3cc(C(C)(C)C)c(C(=S)NCl)c4ccc(c1)c2c34</smiles>

3k (91\%)<smiles>CC(C)(C)c1cc2ccc3cc(C(C)(C)C)c(C(=S)NCc4ccccc4)c4ccc(c1)c2c34</smiles>

3I $(87 \%)$<smiles></smiles>

$3 \mathrm{~m}(82 \%)$

1 - and 4-isomers

(3:1)<smiles>CCCCNC(=O)c1c(C(C)(C)C)cc2ccc3cc(C(C)(C)C)cc4ccc1c2c34</smiles>

3n $(89 \%)$

1 - and 4-isomers

$(6: 1)$

Scheme 1: Triflic acid promoted reaction of 2 with iso(thio)cyanates.

with acetic acid and trifluoroacetic anhydride, (TFAA)/TfOH, In the ${ }^{1} \mathrm{H}$ NMR spectrum of $\mathbf{6}$ we can see two pairs of doublets according to our protocol used for the acylation of pyrene with alkynoic acids [25]. However, in this case we observed exclusive formation of C-4 substituted ketone 6 (Scheme 3 ). split with $J_{\text {meta }}(1.8 \mathrm{~Hz}$ ), assignable to $\mathrm{H}(1), \mathrm{H}(3)$ and $\mathrm{H}(6), \mathrm{H}(8)$ and a pair of doublets split with $J_{\text {ortho }}(9 \mathrm{~Hz})$ assignable to $\mathrm{H}(9), \mathrm{H}(10$ as well as a singlet (H5). 
<smiles>CC(C)(C)c1cc2ccc3cc(C(C)(C)C)cc4ccc(c1)c2c34</smiles>

2

SCN-COOEt $\mathrm{TfOH}$ $\mathrm{CH}_{2} \mathrm{Cl}_{2}, \mathrm{rt}$

30<smiles>CC(C)(C)c1cc2ccc3cc(C(C)(C)C)c(C(=S)NC(=O)[O-])c4ccc(c1)c2c34</smiles><smiles>CCOC(=O)NC(=S)c1c(C(C)(C)C)cc2ccc3cc(C(C)(C)C)c(C(=S)NC(=O)OCC)c4ccc1c2c34</smiles>

4<smiles>CC(C)(C)c1cc2ccc3cc(C(C)(C)C)c(C#N)c4ccc(c1)c2c34</smiles>

5

(main regioisomer)

Scheme 2: Triflic acid promoted reaction of $\mathbf{2}$ with ethoxycarbonyl isothiocyanate

Table 1: Reaction of 2 with ethoxycarbonyl isothiocyanate (2 equiv) and $\mathrm{TfOH}$ (4 equiv) in $\mathrm{CH}_{2} \mathrm{Cl}_{2}$ at room temperature.

\begin{tabular}{ccccc} 
Entry & Reaction time & 30 (\%) & $\mathbf{4}(\%)$ & $\mathbf{5}(\%)$ \\
\hline 1 & $10 \mathrm{~min}$ & 78 & 9 & 7 \\
\hline 3 & $2 \mathrm{~h}$ & 46 & 17 & 13 \\
\hline 4 & $4 \mathrm{~h}$ & 40 & 21 & 18 \\
\hline 5 & $6 \mathrm{~h}$ & 39 & 33 & 20 \\
\hline 6 & $8 \mathrm{~h}$ & 36 & 30 & 21 \\
\hline 7 & $16 \mathrm{~h}$ & 29 & 26 & 32 \\
\hline 8 & 3 days & 15 & 17 & 38 \\
\hline 9 & 7 days & 10 & 14 & 54 \\
70 & $\begin{array}{c}7 \text { days } \\
\text { 12 days }\end{array}$ & 9 & 3 & 43 \\
\hline
\end{tabular}

a3 Equivalents of isothiocyanate were used.

In our opinion, the observed difference in the regioselectivity of the (thio)carbamoylation and acylation of $\mathbf{2}$ may be due to different bulkiness of the reacting electrophile: the electrophilic center of the protonated iso(thio)cyanate is relatively unhindered and able to attack the electronically activated but sterically hindered pyrene 1-position, whereas the bulkier protonated acetyl trifluoroacetate (the postulated electrophile in the examined Friedel-Crafts acylation) attacks sterically the less hindered 4-position.

\section{Conclusion}

We found that triflic acid-promoted (thio)carbamoylation of 2 with aliphatic iso(thio)cyanates occurs selectively at the 1- position, whereas Friedel-Crafts acylation promoted by this acid

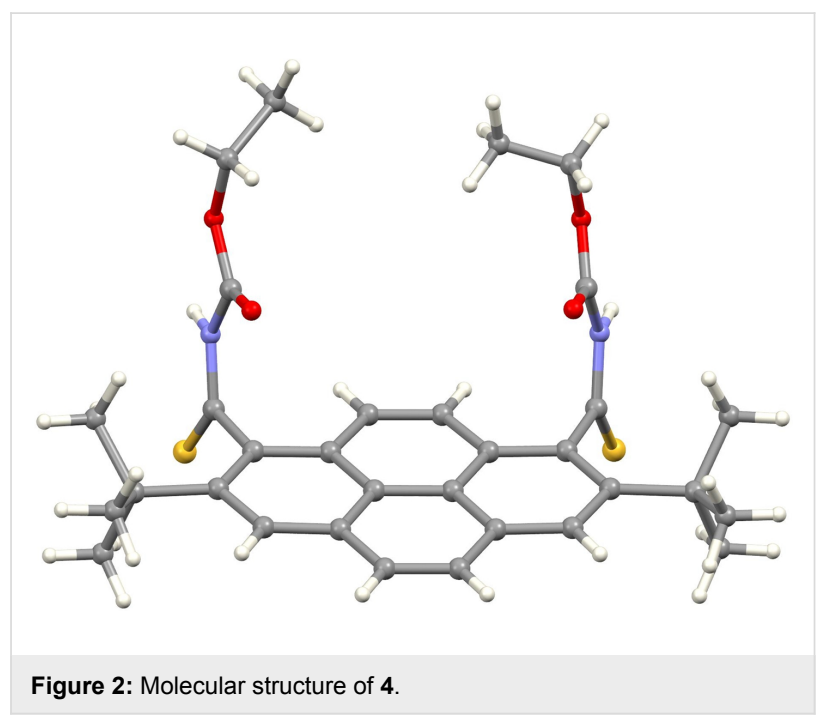

leads to 4-substitution. The reaction is less regioselective for aromatic iso(thio)cyanates. We believe that the described reaction opens up new synthetic routes to novel fluorophores having a sterically hindered pyrene framework.
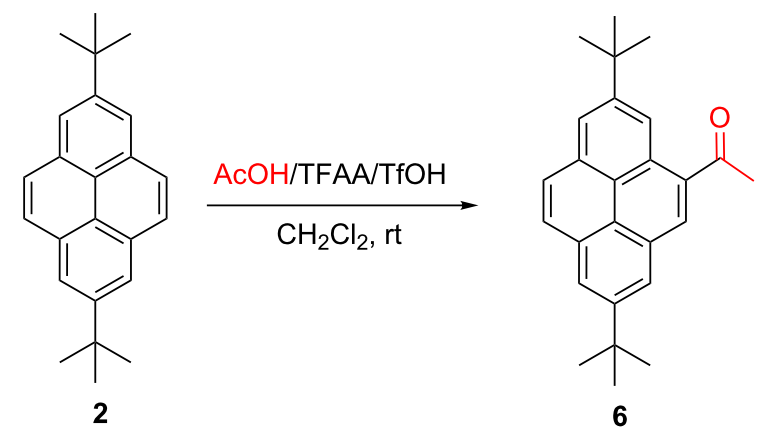

Scheme 3: Friedel-Crafts acylation of 2. 


\section{Experimental General}

All reagents were purchased from Sigma-Aldrich and used without further purification. Solvents were purified before use by reported methods. Column chromatography was carried out on silica gel $60\left(0.040-0.063 \mathrm{~mm}, 230-400\right.$ mesh, Fluka). ${ }^{1} \mathrm{H}$ and ${ }^{13} \mathrm{C}$ NMR spectra were recorded in $\mathrm{CDCl}_{3}$ at $600 \mathrm{MHz}$ for ${ }^{1} \mathrm{H}$ and $150 \mathrm{MHz}$ for ${ }^{13} \mathrm{C}$ at room temperature. Chemical shifts are reported in ppm and are referenced relative to solvent signals.

\section{2,7-Di-tert-butylpyrene-1-carboxamides. General procedure}

Analogous as described in [12]. Isocyanate $(1.2 \mathrm{mmol})$ and TfOH ( $348 \mu \mathrm{L}, 4 \mathrm{mmol})$ were added to a solution of 2,7-di-tertbutylpyrene $(2,314 \mathrm{mg}, 1 \mathrm{mmol})$ in $\mathrm{CH}_{2} \mathrm{Cl}_{2}(10 \mathrm{~mL})$ at room temperature. After stirring for $5 \mathrm{~min}$, the reaction mixture was poured into ice-water $(50 \mathrm{~mL})$ and extracted several times with $\mathrm{CH}_{2} \mathrm{Cl}_{2}$. The combined extracts were dried over anhydrous $\mathrm{Na}_{2} \mathrm{SO}_{4}$ and evaporated. The products were isolated by flash chromatography (eluent: $\mathrm{CH}_{2} \mathrm{Cl}_{2}$ ).

2,7-Di-tert-butyl- $N$-ethylpyrene-1-carboxamide (3a). White solid (293 mg, 76\%). Mp 258-259 ${ }^{\circ} \mathrm{C} ;{ }^{1} \mathrm{H}$ NMR (600 MHz, $\left.\mathrm{CDCl}_{3}\right) \delta 8.26(\mathrm{~s}, 1 \mathrm{H}), 8.20(\mathrm{~d}, J=1.8 \mathrm{~Hz}, 1 \mathrm{H}), 8.18(\mathrm{~d}, J=1.8$ $\mathrm{Hz}, 1 \mathrm{H}), 8.03(\mathrm{~m}, 3 \mathrm{H}), 7.98(\mathrm{~d}, J=9.0 \mathrm{~Hz}, 1 \mathrm{H}), 5.87$ (t, $J=5.4$ $\mathrm{Hz}, 1 \mathrm{H}), 3.74(\mathrm{~m}, 1 \mathrm{H}), 3.64(\mathrm{~m}, 1 \mathrm{H}), 1.67(\mathrm{~s}, 9 \mathrm{H}), 1.58(\mathrm{~s}, 9 \mathrm{H})$ $1.36(\mathrm{t}, J=7.2 \mathrm{~Hz}, 3 \mathrm{H}) ;{ }^{13} \mathrm{C}$ NMR $\left(150 \mathrm{MHz}, \mathrm{CDCl}_{3}\right) \delta 171.8$ 149.2, 144.0, 131.3, 131.1, 130.9, 130.3, 128.7, 128.4, 128.2, $127.2,124.4,123.6,122.7,122.7,122.4,122.4,37.1$, 35.2, 35.1, $32.3,31.9,14.4$; IR (KBr, cm $\left.{ }^{-1}\right)$ : 3414, 3259, 2958, 1629, 1534 1300, 1224, 880; anal. calcd. for $\mathrm{C}_{27} \mathrm{H}_{31} \mathrm{NO}$ : C, 84.11; $\mathrm{H}, 8.10$; N, 3.63; O, 4.15; found: C, 84.07; H, 8.12; N, 3.71 .

(For characterization data of other synthesized amides, see Supporting Information File 1).

\section{2,7-Di-tert-butylpyrene-1-carbothioamides. General procedure}

Analogous as described in [12]. Isothiocyanate $(1.2 \mathrm{mmol})$ and TfOH ( $348 \mu \mathrm{L}, 4 \mathrm{mmol})$ were added to a solution of 2,7-di-tertbutylpyrene $\left(314 \mathrm{mg}, 1 \mathrm{mmol}\right.$, ) in $\mathrm{CH}_{2} \mathrm{Cl}_{2}(10 \mathrm{~mL})$ at room temperature. After stirring for $30 \mathrm{~min}$, the reaction mixture was poured into ice-water $(50 \mathrm{~mL})$ and extracted several times with $\mathrm{CH}_{2} \mathrm{Cl}_{2}$. The combined extracts were dried over anhydrous $\mathrm{Na}_{2} \mathrm{SO}_{4}$ and evaporated. For the reaction with ethoxycarbonyl isothiocyanate, chromatography afforded two fractions. The less polar fraction contained monothioamide 30 and nitrile $\mathbf{5}$, whereas the more polar fraction contained dithioamides $\mathbf{4}$. The separation of $\mathbf{3 o}$ and $\mathbf{5}$ required the second chromatography with $\mathrm{CH}_{2} \mathrm{Cl}_{2}$ /hexanes $(1: 1)$ as the eluent. The yields of 3o, $\mathbf{4}$ and $\mathbf{5}$ are gathered in Table 1.

2,7-Di-tert-butyl- $\boldsymbol{N}$-ethylpyrene-1-carbothioamide (3f). White solid (378 mg, 94\%). Mp $222-223{ }^{\circ} \mathrm{C}$; ${ }^{1} \mathrm{H}$ NMR $\left(600 \mathrm{MHz}, \mathrm{CDCl}_{3}\right) \delta 8.28(\mathrm{~s}, 1 \mathrm{H}), 8.19(\mathrm{~d}, J=1.2 \mathrm{~Hz}, 1 \mathrm{H}), 8.17$ $(\mathrm{d}, J=1.2 \mathrm{~Hz}, 1 \mathrm{H}), 8.08(\mathrm{~d}, J=9.6 \mathrm{~Hz}, 1 \mathrm{H}), 8.02(\mathrm{~d}, J=9.0$ $\mathrm{Hz}, 1 \mathrm{H}), 8.01(\mathrm{~d}, J=9.0 \mathrm{~Hz}, 1 \mathrm{H}), 7.95$ (d, $J=9.0 \mathrm{~Hz}, 1 \mathrm{H}), 7.59$ $(\mathrm{s}, 1 \mathrm{H}), 4.09(\mathrm{~m}, 1 \mathrm{H}), 3.96(\mathrm{~m}, 1 \mathrm{H}), 1.72(\mathrm{~s}, 9 \mathrm{H}), 1.58(\mathrm{~s}, 9 \mathrm{H})$, $1.43(\mathrm{t}, J=7.2 \mathrm{~Hz}, 3 \mathrm{H}) ;{ }^{13} \mathrm{C} \mathrm{NMR}\left(150 \mathrm{MHz}, \mathrm{CDCl}_{3}\right) \delta 203.4$, $149.2,142.5,137.1,131.0,130.9,130.3,128.4,128.0,127.7$, $127.2,124.3,124.2,122.9,122.7,122.3,41.0,37.8,35.2,32.6$, 31.9, 12.8; IR (KBr, cm $\left.{ }^{-1}\right): 3424,3164,2961,1603,1540$, 1391, 1334, 1223, 881; anal. calcd. for $\mathrm{C}_{27} \mathrm{H}_{31} \mathrm{NS}$ : C, 80.75; $\mathrm{H}$, 7.78; N, 3.49; S, 7.98; found: C, 80.70; H, 7.80; N, 3.51, S, 7.99 .

Ethyl (2,7-di-tert-butylpyrene-1-carbonothioyl)carbamate (3o). Yellow solid. Mp 97-98 ${ }^{\circ} \mathrm{C}$; ${ }^{1} \mathrm{H}$ NMR (600 MHz, $\mathrm{CDCl}_{3}$ ) $\delta 9.82(\mathrm{~s}, 1 \mathrm{H}), 8.33(\mathrm{~s}, 1 \mathrm{H}), 8.19(\mathrm{~d}, J=13.2 \mathrm{~Hz}, 2 \mathrm{H}), 8.05$ (s, 2H), $7.99(\mathrm{~m}, 2 \mathrm{H}), 3.92$ (q, $J=7.2 \mathrm{~Hz}, 2 \mathrm{H}), 1.74(\mathrm{~m}, 9 \mathrm{H}), 1.59$ $(\mathrm{m}, 9 \mathrm{H}), 0.97$ (t, $J=7.2 \mathrm{~Hz}, 3 \mathrm{H}) ;{ }^{13} \mathrm{C} \mathrm{NMR}\left(150 \mathrm{MHz}, \mathrm{CDCl}_{3}\right)$ $\delta 211.0,149.0,148.9,141.9,134.9,131.0,130.8,130.2,128.4$, $127.8,127.4,125.9,124.4,123.4,122.7,122.6,122.5,122.2$, 63.0, 37.6, 35.2, 32.5, 31.9, 13.8; IR (KBr, $\left.\mathrm{cm}^{-1}\right)$ : 3447, 3383, 2961, 2906, 2868, 1769, 1479, 1227, 1137, 1043, 881; anal. calcd. for $\mathrm{C}_{28} \mathrm{H}_{31} \mathrm{NO}_{2} \mathrm{~S}$ : C, 75.47; H, 7.01; N, 3.14; O, 7.18; S, 7.20; found: C, 75.34; H, 7.18; N, 3.21, S, 7.14.

Diethyl (2,7-di-tert-butyl.pyrene-1,8-dicarbonothioyl)carbamate (4). Yellow solid. Mp 240-241 ${ }^{\circ} \mathrm{C} ;{ }^{1} \mathrm{H}$ NMR (600 MHz, $\left.\mathrm{CDCl}_{3}\right) \delta 9.75(\mathrm{~s}, 1 \mathrm{H}), 9.72(\mathrm{~s}, 1 \mathrm{H}), 8.28(\mathrm{~m}, 2 \mathrm{H}), 8.02(\mathrm{~s}, 2 \mathrm{H})$, $7.95(\mathrm{~s}, 2 \mathrm{H}), 3.90(\mathrm{~m}, 4 \mathrm{H}), 1.69(\mathrm{~m}, 18 \mathrm{H}), 0.97(\mathrm{~m}, 3 \mathrm{H}), 0.88$ $(\mathrm{m}, 3 \mathrm{H})$. The ${ }^{13} \mathrm{C}$ NMR spectrum was not measured due to limited solubility of this compound. IR $\left(\mathrm{KBr}, \mathrm{cm}^{-1}\right)$ : 3432 , 3385, 3162, 2964, 2910, 1773, 1492, 1228, 1168, 1149, 1110, 1038; anal. calcd. for $\mathrm{C}_{32} \mathrm{H}_{34} \mathrm{~N}_{2} \mathrm{O}_{4} \mathrm{~S}_{2}$ : C, 66.64; $\mathrm{H}, 6.29$; N, 4.86; O, 11.10; S, 11.12; found: C, 66.59; H, 6.33; N, 4.81, S, 11.03 .

(For characterization data of other synthesized thioamides, see Supporting Information File 1).

2,7-Di-tert-butylpyrene-1-carbonitrile (5). Yellow solid. Mp $176-177{ }^{\circ} \mathrm{C} ;{ }^{1} \mathrm{H}$ NMR $\left(600 \mathrm{MHz}, \mathrm{CDCl}_{3}\right) \delta 8.50$ (d, $J=9.0$ $\mathrm{Hz}, 1 \mathrm{H}), 8.30$ (d, $J=1.2 \mathrm{~Hz}, 1 \mathrm{H}), 8.28$ (d, $J=1.2 \mathrm{~Hz}, 1 \mathrm{H}), 8.18$ $(\mathrm{d}, J=9.6 \mathrm{~Hz}, 1 \mathrm{H}), 8.17(\mathrm{~s}, 1 \mathrm{H}), 8.14(\mathrm{~d}, J=9.0 \mathrm{~Hz}, 1 \mathrm{H}), 7.98$ $(\mathrm{d}, J=9.0 \mathrm{~Hz}, 1 \mathrm{H}), 1.81(\mathrm{~s}, 9 \mathrm{H}), 1.62(\mathrm{~s}, 9 \mathrm{H}) ;{ }^{13} \mathrm{C}$ NMR $(150$ $\left.\mathrm{MHz}, \mathrm{CDCl}_{3}\right) \delta 150.9,149.9,134.7,133.4,130.55,130.53$, $130.50,130.0,126.9,124.0,124.0,123.9,122.1,121.9,121.5$, $119.8,103.9,36.2,35.3,31.8,30.8$; IR $\left(\mathrm{KBr}, \mathrm{cm}^{-1}\right): 2958$, 
2905, 2872, 2198, 1600, 1465, 1364, 1228, 879, 733; anal. calcd. for $\mathrm{C}_{25} \mathrm{H}_{25} \mathrm{~N}$ : C, 88.45; H, 7.42; N, 4.13; found: C, 88.39; $\mathrm{H}, 7.51 ; \mathrm{N}, 4.06$.

4-Acetyl-2,7-di-tert-butylpyrene (6). 2,7-Di-tert-butylpyrene $(2,628 \mathrm{mg}, 2 \mathrm{mmol})$ and triflic acid $(174 \mu \mathrm{L}, 2 \mathrm{mmol})$ were added at $0{ }^{\circ} \mathrm{C}$ to a solution of acetic acid $(126 \mu \mathrm{L}, 2.2 \mathrm{mmol})$ and trifluoroacetic anhydride $(278 \mu \mathrm{L}, 2 \mathrm{mmol})$ in $\mathrm{CH}_{2} \mathrm{Cl}_{2}$ $(20 \mathrm{~mL})$. The reaction mixture was warmed to room temperature and stirred for $2 \mathrm{~h}$. After this time the reaction mixture was poured into ice-water $(50 \mathrm{~mL})$ and extracted several times with $\mathrm{CH}_{2} \mathrm{Cl}_{2}$. The combined extracts were dried over anhydrous $\mathrm{Na}_{2} \mathrm{SO}_{4}$ and evaporated. Flash chromatography (eluent: $\mathrm{CH}_{2} \mathrm{Cl}_{2}$ ) afforded the product. Yellow solid. (310 mg, 87\%). Mp 121-122 ${ }^{\circ} \mathrm{C} ;{ }^{1} \mathrm{H}$ NMR (600 MHz, $\left.\mathrm{CDCl}_{3}\right)$ $\delta 9.23(\mathrm{~d}, J=1.8 \mathrm{~Hz}, 1 \mathrm{H}), 8.59(\mathrm{~s}, 1 \mathrm{H}), 8.29(\mathrm{~d}, J=1.2 \mathrm{~Hz}$, $1 \mathrm{H}), 8.28(\mathrm{~d}, J=1.8 \mathrm{~Hz}, 1 \mathrm{H}), 8.24(\mathrm{~d}, J=1.2 \mathrm{~Hz}, 1 \mathrm{H}), 8.05(\mathrm{~d}$, $J=9.0 \mathrm{~Hz}, 1 \mathrm{H}), 8.01(\mathrm{~d}, J=9.0 \mathrm{~Hz}, 1 \mathrm{H}), 2.94(\mathrm{~s}, 3 \mathrm{H}), 1.60(\mathrm{~s}$, $18 \mathrm{H}) ;{ }^{13} \mathrm{C}$ NMR $\left(150 \mathrm{MHz}, \mathrm{CDCl}_{3}\right) \delta 201.9,149.51,149.07$, $134.8,132.3,130.80,130.78,128.9,128.3,127.1,126.7,124.5$, $123.9,123.6,123.5,122.9,121.9,35.5,35.2,31.93,31.88$, 29.9; IR (KBr, cm $\left.{ }^{-1}\right): 2958,2901,2870,1666,1601,1391$, 1225, 1200, 892; anal. calcd. for $\mathrm{C}_{26} \mathrm{H}_{28} \mathrm{O}$ : C, 87.60; H, 7.98; O, 4.48; found: C, 87.57; H, 8.05.

\section{Supporting Information}

\section{Supporting Information File 1}

Characterization data, copies of ${ }^{1} \mathrm{H},{ }^{13} \mathrm{C}$ NMR and IR spectra for synthesized compounds, and crystallographic structure and refinement data of $\mathbf{4}$.

[http://www.beilstein-journals.org/bjoc/content/ supplementary/1860-5397-13-102-S1.pdf]

\section{Supporting Information File 2}

Crystallographic data for 4 .

[http://www.beilstein-journals.org/bjoc/content/ supplementary/1860-5397-13-102-S2.cif]

\section{References}

1. Feng, X.; Hu, J.-Y.; Redshaw, C.; Yamato, T. Chem. - Eur. J. 2016, 22, 11898-11916. doi:10.1002/chem.201600465

2. Figueira-Duarte, T. M.; Müllen, K. Chem. Rev. 2011, 111, 7260-7314. doi:10.1021/cr100428a

3. Casas-Solvas, J. M.; Howgego, J. D.; Davis, A. P. Org. Biomol. Chem. 2014, 12, 212-232. doi:10.1039/С3ОВ41993B

4. Mateo-Alonso, A. Chem. Soc. Rev. 2014, 43, 6311-6324. doi:10.1039/C4CS00119B

5. Niko, Y.; Cho, Y.; Kawauchi, S.; Konishi, G.-i. RSC Adv. 2014, 4, 36480-36484. doi:10.1039/C4RA06282E
6. Piotrowicz, M.; Zakrzewski, J.; Métivier, R.; Brosseau, A.; Makal, A.; Woźniak, K. J. Org. Chem. 2015, 80, 2573-2581. doi:10.1021/jo502619k

7. Chercka, D.; Yoo, S.-J.; Baumgarten, M.; Kim, J.-J.; Müllen, K. J. Mater. Chem. C 2014, 2, 9083-9086. doi:10.1039/C4TC01801J

8. Rodenburg, L.; Brandsma, R.; Tintel, C.; van Thuijl, J.; Lugtenburg, J.; Cornelisse, J. Recl. Trav. Chim. Pays-Bas 1986, 105, 156-161. doi:10.1002/recl.19861050504

9. Yamato, T.; Fujimoto, M.; Miyazawa, A.; Matsuo, K. J. Chem. Soc., Perkin Trans. 11997, 1201-1208. doi:10.1039/a606200h

10. Bock, H.; Subervie, D.; Mathey, P.; Pradhan, A.; Sarkar, P.; Dechambenoit, P.; Hillard, E. A.; Durola, F. Org. Lett. 2014, 16, 1546-1549. doi:10.1021/ol500154k

11. Wrona-Piotrowicz, A.; Zakrzewski, J.; Métivier, R.; Brosseau, A.; Makal, A.; Woźniak, K. RSC Adv. 2014, 4, 56003-56012. doi:10.1039/C4RA07045C

12. Wrona-Piotrowicz, A.; Zakrzewski, J.; Gajda, A.; Gajda, T.; Makal, A.; Brosseau, A.; Métivier, R. Beilstein J. Org. Chem. 2015, 11, 2451-2458. doi:10.3762/bjoc.11.266

13. Ozaki, K.; Kawasumi, K.; Shibata, M.; Ito, H.; Itami, K. Nat. Commun. 2015, 6, No. 6251. doi:10.1038/ncomms7251.

14. Duan, Z.; Hoshino, D.; Yang, Z.; Yano, H.; Ueki, H.; Liu, Y.; Ohuchi, H.; Takayanagi, Y.; Zhao, G.; Nishioka, Y. Mol. Cryst. Liq. Cryst. 2011, 538, 199-207. doi:10.1080/15421406.2011.564018

15. Gonell, S.; Poyatos, M.; Peris, E. Chem. - Eur. J. 2014, 20 , 9716-9724. doi:10.1002/chem.201304952

16. García, R.; More, S.; Melle-Franco, M.; Mateo-Alonso, A. Org. Lett. 2014, 16, 6096-6099. doi:10.1021/ol5029332

17. Paudel, A.; Hu, J.-Y.; Yamato, T. J. Chem. Res., Synop. 2008, 457-460. doi:10.3184/030823408X338710

18. Jang, K.; Ranasinghe, A. D.; Heske, C.; Lee, D.-C. Langmuir 2010, 26, 13630-13636. doi:10.1021/la101921c

19. Mochida, K.; Kawasumi, K.; Segawa, Y.; Itami, K. J. Am. Chem. Soc. 2011, 133, 10716-10719. doi:10.1021/ja202975w

20. Jiang, L.; Papageorgiou, A. C.; Oh, S. C.; Sağlam, Ö.; Reichert, J.; Duncan, D. A.; Zhang, Y.-Q.; Klappenberger, F.; Guo, Y.; Allegretti, F.; More, S.; Bhosale, R.; Mateo-Alonso, A.; Barth, J. V. ACS Nano 2016, 10, 1033-1041. doi:10.1021/acsnano.5b06340

21. Jagodziński, T. S. Chem. Rev. 2003, 103, 197-228. doi:10.1021/cr0200015

22. Volkov, A.; Tinnis, F.; Slagbrand, T.; Trillo, P.; Adolfsson, H. Chem. Soc. Rev. 2017, 45, 6685-6697. doi:10.1039/C6CS00244G

23. Zhu, R.-Y.; Farmer, M. E.; Chen, Y.-Q.; Yu, J.-Q. Angew. Chem., Int. Ed. 2016, 55, 10578-10599. doi:10.1002/anie.201600791

24. Yamaguchi, K.; Yajima, K.; Mizuno, N. Chem. Commun. 2012, 48, 11247-11249. doi:10.1039/c2cc36635e

25. Flamholc, R.; Plażuk, D.; Zakrzewski, J.; Métivier, R.; Nakatani, K.; Makal, A.; Woźniak, K. RSC Adv. 2014, 4, 31594-31601. doi:10.1039/C4RA03961K 


\section{License and Terms}

This is an Open Access article under the terms of the Creative Commons Attribution License

(http://creativecommons.org/licenses/by/4.0), which permits unrestricted use, distribution, and reproduction in any medium, provided the original work is properly cited.

The license is subject to the Beilstein Journal of Organic Chemistry terms and conditions:

(http://www.beilstein-journals.org/bjoc)

The definitive version of this article is the electronic one which can be found at:

doi:10.3762/bjoc. 13.102 